\title{
Admissibility and Exact Observability of Observation Operators for Semigroups
}

\author{
JONATHAN R. PARTINGTON AND SANDRA POTT
}

ABstract. A characterization of the admissibility of an observation operator for a linear semigroup system in terms of certain rational functions of the infinitesimal generator is given, extending work of Grabowski-Callier and $\mathrm{GaO}-\mathrm{Hu}$. The same functions are then used to give new necessary and sufficient conditions for admissibility and exact observability, in both infinite and finite time. In the special case of the right shift semigroup on $L^{2}((0, \infty), \mathcal{K})$, where $\mathcal{K}$ is a Hilbert space, this translates into necessary and sufficient conditions for boundedness of vectorial Hankel operators, including a formulation in terms of test functions. This leads finally to a characterization of operator-valued functions in the dual of trace-class valued $H^{1}$ spaces in terms of BMO-type conditions.

\section{INTRODUCTION}

In this paper we consider a linear system given by the equations

$$
\begin{aligned}
\dot{x}(t) & =A x(t), \quad t \geq 0, \\
y(t) & =C x(t),
\end{aligned}
$$

where $A$ is the infinitesimal generator of a $C_{0}$-semigroup $(T(t))_{t \geq 0}$ on a Hilbert space $\mathcal{H}$ (the state space) and $C$, an observation operator for $(T(t))_{t \geq 0}$, is assumed to be a linear bounded operator from $\mathcal{D}(A)$, the domain of $A$, to another Hilbert space $Y$ (the output space). Here $\mathcal{D}(A)$ is given the graph norm $\|x\|_{g}=\left(\|x\|^{2}+\|A x\|^{2}\right)^{1 / 2}$.

A mild solution of $\dot{x}(t)=A x(t)$ with initial condition $x(0)=$ $x_{0} \in \mathcal{H}$ means the continuous function $x(t)=T(t) x_{0}$, for $t \geq 0$. An additional assumption is needed to guarantee that the output $y$ of

2000 Mathematics Subject Classification. 47D06, 93B28, 47B35, 32A37.

The second author gratefully acknowledges support by EPSRC. 
the system is in $L^{2}((0, \infty) ; Y)$. In the following we use the notation $\mathcal{L}(X, Y)$ for the set of bounded linear operators from a space $X$ to a space $Y$.

Definition 1.1. Let $C \in \mathcal{L}(D(A), Y)$. Then $C$ is called an (infinitetime) admissible observation operator for $(T(t))_{t \geq 0}$ if there is some $K>0$ such that

$$
\|C T(\cdot) x\|_{L^{2}((0, \infty) ; Y)} \leq K\|x\|, \quad x \in D(A) .
$$

Moreover, $C$ is weakly admissible if the functional $x \mapsto\langle C x, y\rangle$ is admissible for each $y \in Y$.

It is known that admissibility is a strictly stronger notion than weak admissibility $[6,13]$.

There has been much work done towards characterizing admissibility in terms of $A$ and $C$, without knowledge of a precise formula for the semigroup $(T(t))_{t>0}$ - in many PDE situations one knows $A$ but cannot write down $(T(t))_{t \geq 0}$ explicitly - and we refer to the survey [5] and the book [10] for further information and many relevant references. In particular, there is a dual notion of admissibility for control operators, and the results of this paper can be translated into these terms as well.

There is one simple necessary and sufficient condition for admissibility that applies in the case when the output space is finitedimensional, and $(T(t))_{t \geq 0}$ is a contraction semigroup, namely that there is a constant $M>0$ such that

$$
\left\|C(\lambda-A)^{-1}\right\| \leq \frac{M}{(\operatorname{Re} \lambda)^{1 / 2}}
$$

for all $\lambda \in \mathbb{C}_{+}=\{z \in \mathbb{C}: \operatorname{Re} z>0\}$. See $[4,11,12]$ for further information.

A weaker notion than admissibility (but equivalent for systems that are exponentially stable) is the following.

Definition 1.2. Let $C \in \mathcal{L}(D(A), Y)$ and let $\tau>0$. Then $C$ is called a finite-time admissible observation operator for $(T(t))_{t \geq 0}$ if there is some $K>0$ such that

$$
\|C T(\cdot) x\|_{L^{2}((0, \tau) ; Y)} \leq K\|x\|, \quad x \in D(A) .
$$

This definition is independent of $\tau$ (see Section 4).

In this article we also consider various notions of exact observability, which we now define. 
Definition 1.3. Let $C \in \mathcal{L}(D(A), Y)$ and let $\tau>0$. Then $(A, C)$ is exactly observable if there is some $K>0$ such that

$$
\|C T(\cdot) x\|_{L^{2}((0, \infty) ; Y)} \geq K\|x\|, \quad x \in D(A),
$$

and $(A, C)$ is $\tau$-exactly observable if there is some $K>0$ such that

$$
\|C T(\cdot) x\|_{L^{2}((0, \tau) ; Y)} \geq K\|x\|, \quad x \in D(A) .
$$

It is well-known that the notion of $\tau$-exact observability may depend on $\tau$, see Section 5 .

For the case of an exponentially stable semigroup Grabowski and Callier [3] gave a characterization of infinite-time admissibility in terms of an operator-valued analytic function; Gao and Hou [1] extended this result to semigroups such that $\mathbb{C}_{+} \subseteq \rho(A)$. As we shall show, it is possible to characterize other properties of semigroup systems in terms of these operator-valued analytic functions, and we shall refer to such results as $G C G H$ theorems.

In this paper we proceed as follows. In Section 2 we use alternative and arguably somewhat simpler methods to derive a slightly stronger form of the GCGH theorem characterizing infinite-time admissibility. Then in Section 3 we prove a GCGH-type theorem giving a new characterization of exact observability. In Sections 4 and 5 we provide analogous results characterizing finite-time admissibility and exact observability. When the underlying semigroup is the right-shift semigroup on $L^{2}(0, \infty)$, it is known that admissibility is equivalent to the boundedness of certain Hankel operators (see $[6,7,8]$ ), and in Sections 6 and 7 we consider this in the light of the GCGH conditions: thus we have a new characterization of the boundedness of vectorial Hankel operators and, as a consequence, a characterization of operator-valued functions in the dual of $H^{1}\left(\mathbb{C}_{+}, S_{1}\right)$, the Hardy space of analytic functions on $\mathbb{C}_{+}$with values in the trace class $S_{1}$.

We begin by defining the analytic functions that we shall require. For $(T(t))_{t \geq 0}$, a strongly continuous semigroup on $\mathcal{H}$ with generator $A$ such that $\rho(A) \supseteq \mathbb{C}_{+}$, and $C: \mathcal{D}(A) \rightarrow Y$ an observation operator, let

$$
F: \mathbb{C}_{+} \rightarrow \mathcal{L}\left(\mathcal{H}, H^{2}\left(\mathbb{C}_{+}, Y\right)\right), \quad F(\lambda): z \mapsto C(\lambda-A)^{-1} \frac{1}{\lambda+z} .
$$

Thus $F(\lambda)$ is the Laplace transform of the operator-valued function $f(\lambda)$ defined by $t \mapsto e^{-\lambda t} C(\lambda-A)^{-1}$. We also define

$$
\gamma_{n}(\lambda)(t)=\frac{1}{n !} \frac{\partial^{n} f}{\partial \lambda^{n}}(\lambda)(t)
$$


so that

$$
\gamma_{n}(\lambda)(t)=(-1)^{n} \sum_{k=0}^{n} \frac{t^{k} e^{-\lambda t}}{k !} C(\lambda-A)^{-(n-k+1)} .
$$

The following identity will be needed later.

Proposition 1.4. We have

$$
\gamma_{n}(\lambda)(t)=\frac{(-1)^{n}}{n !} \int_{0}^{\infty} C T(w) e^{-\lambda(t+w)}(t+w)^{n} d w
$$

Proof. We begin with the formula

$$
F(\lambda)(z)=\int_{0}^{\infty} e^{-z t} e^{-\lambda t} d t \int_{0}^{\infty} C T(w) e^{-\lambda w} d w
$$

Differentiating with respect to $\lambda$ (such operations are easily seen to be valid for $\lambda, z \in \mathbb{C}_{+}$) we obtain

$$
F^{(n)}(\lambda)(z)=\int_{0}^{\infty} \int_{0}^{\infty} e^{-z t} C T(w) e^{-\lambda(t+w)}(-1)^{n}(t+w)^{n} d t d w,
$$

and thus

$$
f^{(n)}(\lambda)(t)=(-1)^{n} \int_{0}^{\infty} C T(w) e^{-\lambda(t+w)}(t+w)^{n} d w
$$

which implies the result.

Using these functions we are now ready to begin to characterize the admissibility and observability properties associated with the semigroup.

\section{Infinite-time Admissibility}

Notice that for $z, \lambda \in \mathbb{C}_{+}$, we have an operator $F^{(n)}(\lambda)(z): \mathcal{H} \rightarrow Y$ given by $h \mapsto\left(F^{(n)}(\lambda) h\right)(z)$. If $\lambda>K$, then $F^{(n)}(\lambda)(z)$ is actually well-defined for all $z \in\{s \in \mathbb{C}: \operatorname{Re} s>-K\}$.

Lemma 2.1. Let $\mathcal{H}$ be a Hilbert space and $(T(t))_{t \geq 0}$ be a strongly continuous semigroup on $\mathcal{H}$ with generator $A$ such that $\mathbb{C}_{+} \subseteq \rho(A)$. Let $C: \mathcal{D}(A) \rightarrow Y$ be an observation operator, and let $F$ be defined as in (3). Then for each $r>0, h \in \mathcal{H}$,

$$
(-1)^{n} \frac{(r n)^{n+1}}{n !}\left(F^{(n)}(r n)\right)(z) h \longrightarrow C(z+A)^{-1}\left(e^{A / r}-e^{-z / r}\right) h
$$

as $n \rightarrow \infty$ uniformly on compact sets in $\mathbb{C}_{-}$. 
Proof. Note that for given $\lambda \in \mathbb{C}_{+}, F(\lambda)=C(\lambda-A)^{-1} \frac{1}{\lambda+z}$ is welldefined as an analytic function in $z$ on the half-plane

$$
\mathbb{C}_{-\operatorname{Re} \lambda}=\{z \in \mathbb{C}: \operatorname{Re} z>-\operatorname{Re} \lambda\} .
$$

For $\lambda \in \rho(A),-z \in \rho(A)$, and $\lambda+z \neq 0$, we have the identity

$$
F(\lambda)(z)=C(A+z)^{-1}\left[(\lambda-A)^{-1}-(\lambda+z)^{-1}\right],
$$

since on multiplying this expression on the right by $(\lambda-A)(\lambda+z)$ we obtain

$$
C(A+z)^{-1}[(\lambda+z)-(\lambda-A)]=C .
$$

So in particular, (4) holds for

$$
z \in \mathbb{C}_{(-\operatorname{Re} \lambda, 0)}=\{z \in \mathbb{C}: 0>\operatorname{Re} z>-\operatorname{Re} \lambda\} .
$$

Now, choosing a suitably small disc $D$ in $\mathbb{C}_{+}$and differentiating $F$ $n$ times with respect to $\lambda$, we obtain

$$
F^{(n)}(\lambda)(z)=(-1)^{n} n ! C(A+z)^{-1}\left[(\lambda-A)^{-n-1}-(\lambda+z)^{-n-1}\right]
$$

for $z \in \mathbb{C}_{\left(-\frac{1}{2} \operatorname{Re} \lambda, 0\right)}$. Putting $\lambda=r n$, we find that for each $z \in \mathbb{C}_{-}$,

$$
\begin{aligned}
(-1)^{n} \frac{(r n)^{n+1}}{n !} & \left(F^{(n)}(r n)\right)(z)= \\
& \quad C(-A-z)^{-1}\left(\left(\frac{n r}{n r+z}\right)^{n+1}-\left(\mathbf{1}-\frac{1}{n r} A\right)^{-(n+1)}\right),
\end{aligned}
$$

for suitably large $n$. The right hand side in this equation is easily seen to converge to $C(z+A)^{-1}\left(e^{A / r}-e^{-z / r}\right)$ in the strong operator topology (SOT). Furthermore, for any given $h \in \mathcal{H}$, the norm convergence in $Y$ of

$$
(-1)^{n} \frac{(r n)^{n+1}}{n !}\left(F^{(n)}(r n) h\right)(z) \longrightarrow C(A+z)^{-1}\left(e^{A / r}-e^{-z / r}\right) h
$$

is uniform in $z$ on each compact subset of $\mathbb{C}_{-}$.

Before we go on to prove a stronger form of the GCGH theorem, we note the following lemma.

Lemma 2.2. For all $\left.n \in \mathbb{N}, h \in \mathcal{H}, g \in L^{2}((0, \infty), Y)\right)$,

$$
\left|\left\langle\gamma_{n}(\lambda) h, g\right\rangle_{L^{2}((0, \infty), Y)}\right| \leq \frac{1}{\lambda^{n+1}}\|C T(\cdot) h\|_{L^{2}((0, \infty), Y)}\|g\| .
$$


Proof. From Proposition 1.4, we have

$$
\gamma_{n}(\lambda)(t)=\frac{(-1)^{n}}{n !} \int_{0}^{\infty} C T(w) e^{-\lambda(t+w)}(t+w)^{n} d w .
$$

Take $h \in \mathcal{H}$ and $g \in L^{2}((0, \infty), Y)$. Then, writing $t+w=z$ we get

$$
\begin{aligned}
& \left\langle\gamma_{n}(\lambda) h, g\right\rangle_{L^{2}((0, \infty), Y)}= \\
& \quad \frac{(-1)^{n}}{n !} \int_{z=0}^{\infty} z^{n} e^{-\lambda z} d z \int_{w=0}^{z}\langle C T(w) h, g(z-w)\rangle_{Y} d w .
\end{aligned}
$$

Hence, since $\int_{0}^{\infty} z^{n} e^{-\lambda z} d z=n ! / \lambda^{n+1}$, this proves the lemma.

Theorem 2.3 (see [3, Theorem 2.3] and [1, Theorem 1]). Let $\mathcal{H}$ be a Hilbert space and $(T(t))_{t>0}$ be a strongly continuous semigroup on $\mathcal{H}$ with generator $A$ such that $\rho(A) \supseteq \mathbb{C}_{+}$. Let $C: \mathcal{D}(A) \rightarrow Y$ be an observation operator. Then $(A, C)$ is (infinite-time) admissible if and only if there exists $M, K, N_{0}>0$ such that

$$
\left\|\gamma_{n}(\lambda)\right\|_{\mathcal{L}\left(\mathcal{H}, L^{2}((0, \infty), Y)\right)} \leq \frac{M}{\lambda^{n+1}} \quad\left(\lambda \in \mathbb{R}_{+}, \lambda>K, n>N_{0}\right)
$$

where $\gamma_{n}(\lambda)(t)=(-1)^{n} \sum_{k=0}^{n} \frac{t^{k} e^{-\lambda t}}{k !} C(\lambda-A)^{-(n-k+1)}$ for $t \geq 0$.

Proof. Taking Laplace transforms, we obtain that

$$
\left\|\gamma_{n}(\lambda) h\right\|_{L^{2}((0, \infty), Y)}=\left\|\frac{1}{n !} F^{(n)}(\lambda) h\right\|_{H^{2}\left(\mathbb{C}_{+}, Y\right)}
$$

for all $\lambda \in \mathbb{R}, n \in \mathbb{N}, h \in \mathcal{H}$.

By Lemma 2.2, we obtain the necessity of condition (6) for all $n \in \mathbb{N}, \lambda \in \mathbb{R}_{+}$.

Conversely, suppose that (6) holds. By Lemma 2.1, we obtain that

$$
\int_{-\infty}^{\infty}\left\|C(i \omega-\delta+A)^{-1}\left(e^{A / r}-e^{-(i \omega-\delta) / r}\right) h\right\|^{2} \leq M\|h\|^{2}
$$

for all $h \in \mathcal{H}, \delta>0$. It follows that $C(z+A)^{-1}\left(e^{A / r}-e^{-z / r}\right) h \in$ $H^{2}\left(\mathbb{C}_{-}, Y\right)$ for each $r>0$. Taking Laplace transforms again, we see that

$$
\begin{aligned}
C(A+z)^{-1} & \left(e^{A / r}-e^{-z / r}\right) h \\
& =C(A+z)^{-1}\left(e^{(A+z) / r}-1\right) e^{-z / r} h \\
& =\mathcal{L}\left(\chi_{[0,1 / r]} T(\cdot) h\right)(-z) e^{-z / r} \quad\left(z \in \mathbb{C}_{-}\right)
\end{aligned}
$$


and therefore

$$
\begin{array}{rl}
\int_{0}^{1 / r}\|C T(t) h\|^{2} & d t \\
& =\left\|C(A+z)^{-1}\left(e^{A / r}-e^{-z / r}\right) e^{z / r} h\right\|_{H^{2}\left(\mathbb{C}_{-}, Y\right)} \\
& =\left\|C(A+z)^{-1}\left(e^{A / r}-e^{-z / r}\right) h\right\|_{H^{2}\left(\mathbb{C}_{-}, Y\right)} \leq M^{2}\|h\|^{2}
\end{array}
$$

for each $r>0$. It follows that $(A, C)$ is admissible with admissibility constant $M$.

We use the seemingly weaker condition

$$
\left\|\gamma_{n}(\lambda)\right\|_{\mathcal{L}\left(\mathcal{H}, L^{2}((0, \infty), Y)\right)} \leq \frac{M}{\lambda^{n+1}} \quad\left(\lambda \in \mathbb{R}_{+}, \lambda>K, n>N_{0}\right)
$$

than in [1], where the inequality is required for all $n \in \mathbb{N}, \lambda>0$. However, it is easily seen that our condition at once implies the corresponding inequality for all $n \in \mathbb{N}, \lambda>0$.

Proposition 2.4. Let $(T(t))_{t>0}$ be a strongly continuous semigroup on $\mathcal{H}$ with generator $A$ such that $\rho(A) \supseteq \mathbb{C}_{+}$, let $C: \mathcal{D}(A) \rightarrow Y$ be an observation operator, and let $F$ be as above. Suppose that there exist constants $M$ and $K$ such that for each $n \geq 0$ and for each $\lambda \in \mathbb{R}$ with $\lambda>K$

$$
\left\|F^{(n)}(\lambda)\right\|_{\mathcal{L}\left(H, H^{2}\left(\mathbb{C}_{+}, Y\right)\right)} \leq n ! \frac{M}{\lambda^{n+1}} .
$$

Then

$$
\left\|F^{(n)}(\lambda)\right\|_{\mathcal{L}\left(H, H^{2}\left(\mathbb{C}_{+}, Y\right)\right)} \leq n ! \frac{M}{(\operatorname{Re} \lambda)^{n+1}} \text { for all } \lambda \in \mathbb{C}_{+}
$$

with the same constant $M$.

Proof. Suppose that (8) holds with certain constants $K, M>0$. Let $\lambda \in \mathbb{C}_{+}$. For sufficiently large $\lambda_{0}>K$, one has $\left|\lambda-\lambda_{0}\right|<\lambda_{0}$. Then, using the Taylor expansion for the analytic operator-valued 
function $F$, we have

$$
\begin{aligned}
\left\|F^{(n)}(\lambda)\right\|_{\mathcal{L}\left(H, H^{2}\left(\mathbb{C}_{+}, Y\right)\right)} & \\
& =\left\|\sum_{k=n}^{\infty} \frac{F^{(k)}\left(\lambda_{0}\right)}{k !} \frac{k !}{(k-n) !}\left(\lambda-\lambda_{0}\right)^{k-n}\right\|_{\mathcal{L}\left(H, H^{2}\left(\mathbb{C}_{+}, Y\right)\right)} \\
& \leq M \sum_{k=n}^{\infty} \frac{k !}{(k-n) ! \lambda_{0}^{k+1}} \lambda_{0}^{k-n}\left(\frac{\left|\lambda-\lambda_{0}\right|}{\lambda_{0}}\right)^{k-n} \\
& =M \frac{1}{\lambda_{0}^{n+1}} \sum_{j=0}^{\infty} \frac{(j+n) !}{j !}\left|\frac{\lambda-\lambda_{0}}{\lambda_{0}}\right|^{j} \\
& \leq M \frac{n !}{\left(\lambda_{0}-\left|\lambda-\lambda_{0}\right|\right)^{n+1}} .
\end{aligned}
$$

Letting $\lambda_{0} \rightarrow+\infty$, we obtain $\left\|F^{(n)}(\lambda)\right\|_{\mathcal{L}\left(H, H^{2}\left(\mathbb{C}_{+}, Y\right)\right)} \leq M \frac{n !}{(\operatorname{Re} \lambda)^{n+1}}$.

Remark 2.5. In particular, for $n=1$ we recover the resolvent condition for all $\lambda \in \mathbb{C}_{+}$.

Remark 2.6. If we assume that $\lim _{\lambda \rightarrow+\infty}\left\|C(\lambda-A)^{-n}\right\|=0$ for all $n \in \mathbb{N}$ (for example, by assuming weak admissibility of $(C, A)$ ), then we obtain the following result by simple integration:

Suppose that there exist constants $N_{0}, M$ and $K$ such that for each $n \geq N_{0}$ and for each $\lambda \in \mathbb{R}$ with $\lambda>K$

$$
\left\|F^{(n)}(\lambda)\right\|_{\mathcal{L}\left(H, H^{2}\left(\mathbb{C}_{+}, Y\right)\right)} \leq n ! \frac{M}{\lambda^{n+1}} .
$$

Then

$$
\left\|F^{(n)}(\lambda)\right\|_{\mathcal{L}\left(H, H^{2}\left(\mathbb{C}_{+}, Y\right)\right)} \leq n ! \frac{M}{(\operatorname{Re} \lambda)^{n+1}}
$$

for all $\lambda \in \mathbb{C}_{+}, n \in \mathbb{N}$ with the same constant $M$.

\section{Exact Observability}

The operator functions $\gamma_{n}$ can also be used to obtain a characterisation of exact observability. Before we state the main theorem, we require some further notation. Recall that $\mathcal{O}(D)$ denotes the set of analytic functions on a domain $D \subseteq \mathbb{C}$. Then for $\gamma>0$, let

$$
H_{-\gamma}^{2}\left(\mathbb{C}_{+}\right)=\left\{\left.f \in \mathcal{O}\left(\mathbb{C}_{+}-\gamma\right)\left|\lim _{\delta \downarrow 0} \int_{-\infty}^{\infty}\right| f(i \omega-\gamma+\delta)\right|^{2} d \omega<\infty\right\} .
$$


This is a (non-closed) subspace of $H^{2}\left(\mathbb{C}_{+}\right)$. Similarly, let

$$
H_{\gamma}^{2}\left(\mathbb{C}_{-}\right)=\left\{\left.f \in \mathcal{O}\left(\mathbb{C}_{-}+\gamma\right)\left|\lim _{\delta \downarrow 0} \int_{-\infty}^{\infty}\right| f(i \omega+\gamma-\delta)\right|^{2} d \omega<\infty\right\},
$$

which is a non-closed subspace of $H^{2}\left(\mathbb{C}_{-}\right)$.

Here is our result about exact observability.

Theorem 3.1. Let $(T(t))_{t \geq 0}$ be a strongly continuous semigroup on $\mathcal{H}$ with generator $A$ such that $\rho(A) \supseteq \mathbb{C}_{+}$, and let $C: \mathcal{D}(A) \rightarrow Y$ be an observation operator. Then $(A, C)$ is exactly observable if and only if there exists a constant $m>0$ such that, for each $h \in \mathcal{H}$, there exist $n \geq 0$ and $\lambda>0$ with

$$
\left\|\gamma_{n}(\lambda) h\right\| \geq m\|h\| / \lambda^{n+1} .
$$

Proof. The "if"-part follows easily from Lemma 2.2, since this implies that

$$
\|C T(\cdot) h\|_{L^{2}((0, \infty), Y)} \geq \lambda^{n+1}\left\|\gamma_{n}(\lambda) h\right\| \quad\left(n \in \mathbb{N}, \lambda \in \mathbb{R}_{+}, h \in \mathcal{H}\right) .
$$

Now suppose that $(A, C)$ is exactly observable with constant $m>0$. Let $\varepsilon>0$. Using the identity (7) again, we find that for each $h \in \mathcal{H}$, there exist $r, \delta>0$ such that

$$
\begin{aligned}
m^{2}\|h\|^{2} & \leq \int_{0}^{\infty}\|C T(t) h\|^{2} d t \\
& \leq(1+\varepsilon) \int_{0}^{1 / r}\|C T(t) h\|^{2} d t \\
& =(1+\varepsilon)\left\|C(A+z)^{-1}\left(e^{A / r}-e^{-z / r}\right) e^{z / r} h\right\|_{H^{2}\left(\mathbb{C}_{-}, Y\right)} \\
& =(1+\varepsilon)\left\|C(A+z)^{-1}\left(e^{A / r}-e^{-z / r}\right) h\right\|_{H^{2}\left(\mathbb{C}_{-}, Y\right)} \\
& \leq(1+\varepsilon)^{2} \int_{-\infty}^{\infty}\left\|C(A+i \omega-\delta)^{-1}\left(e^{A / r}-e^{-(i \omega-\delta) / r}\right)\right\|^{2} d \omega,
\end{aligned}
$$

provided that $\int_{0}^{\infty}\|C T(t) h\|^{2} d t<\infty$. If $\int_{0}^{\infty}\|C T(t) h\|^{2} d t=\infty$, then we can use (7) to make $\int_{-\infty}^{\infty}\left\|C(A+i \omega-\delta)^{-1}\left(e^{A / r}-e^{-(i \omega-\delta) / r}\right)\right\|^{2} d \omega$ as large as we wish. In either case, we can find $\delta, r>0$ such that

$$
\int_{-\infty}^{\infty}\left\|C\left(A+i \omega-\delta^{\prime}\right)^{-1}\left(e^{A / r}-e^{-\left(i \omega-\delta^{\prime}\right) / r}\right) h\right\|^{2} d \omega \geq \frac{m^{2}}{(1+\varepsilon)^{2}}\|h\|^{2}
$$

for $0<\delta^{\prime} \leq \delta$. As $\bigcup_{\gamma>0} H_{\gamma}^{2}\left(\mathbb{C}_{-}, Y\right)$ is dense in $H^{2}\left(\mathbb{C}_{-}, Y\right)$, there are $\gamma>0, g \in H_{\gamma}^{2}\left(\mathbb{C}_{-}, Y\right)$ with $\|g\|_{H^{2}\left(\mathbb{C}_{-}, Y\right)}=1$ and $0<\delta^{\prime}<\min (\gamma, \delta)$ 
such that

$$
\begin{array}{r}
\left|\int_{-\infty}^{\infty}\left\langle C\left(A+i \omega-\delta^{\prime}\right)^{-1}\left(e^{A / r}-e^{-\left(i \omega-\delta^{\prime}\right) / r}\right) h, g\left(i \omega-\delta^{\prime}\right)\right\rangle d \omega\right| \\
\geq \frac{m^{2}}{(1+\varepsilon)^{3}}\|h\|^{2} .
\end{array}
$$

By Lemma 2.1, there exists $n \in \mathbb{N}$ such that

$\frac{m^{2}}{(1+\varepsilon)^{4}}\|h\|^{2} \leq\left|\int_{\infty}^{\infty}\left\langle\frac{(n r)^{n+1}}{n !}\left(F^{(n)}(r n)\right)\left(i \omega-\delta^{\prime}\right) h, g\left(i \omega-\delta^{\prime}\right)\right\rangle d \omega\right|$.

We can assume that $\frac{(n r)^{n+1}}{n !}\left(F^{(n)}(r n)\right) \in H^{2}\left(\mathbb{C}_{+}, Y\right)$. Using the notation $p_{z}(i t)=\operatorname{Re} z \frac{1}{|z+i t|^{2}}, z \in \mathbb{C}_{+}, t \in \mathbb{R}$ for the Poisson kernel of the right half plane, we obtain the identity

$$
\begin{aligned}
\int_{-\infty}^{\infty}\left\langle u\left(i \omega-\delta^{\prime}\right),\right. & \left.v\left(i \omega-\delta^{\prime}\right)\right\rangle d \omega \\
& =\int_{-\infty}^{\infty}\left\langle u\left(i \omega-\delta^{\prime}\right), \int_{-\infty}^{\infty} p_{i \omega+\delta^{\prime}}(i s) v(s) d s\right\rangle d \omega \\
& =\int_{-\infty}^{\infty}\left\langle\int_{-\infty}^{\infty} p_{i s+\delta^{\prime}}(i \omega) u\left(i \omega-\delta^{\prime}\right) d \omega, v(s)\right\rangle d s \\
& =\int_{-\infty}^{\infty}\langle u(i \omega), v(i \omega)\rangle d \omega
\end{aligned}
$$

for $u \in H_{-\gamma}^{2}\left(\mathbb{C}_{+}, Y\right), v \in H_{\gamma}^{2}\left(\mathbb{C}_{-}, Y\right), 0<\delta^{\prime}<\gamma$.

Consequently, with $\lambda=r n$,

$$
\begin{aligned}
\| \lambda^{n+1} \gamma_{n}(\lambda) & h \| \\
& =\left\|\frac{(n r)^{n+1}}{n !}\left(F^{(n)}(r n)\right) h\right\| \\
& \geq\left|\int_{\infty}^{\infty}\left\langle\frac{(n r)^{n+1}}{n !}\left(F^{(n)}(r n)\right)(i \omega) h, g(i \omega)\right\rangle d \omega\right| \\
& =\left|\int_{\infty}^{\infty}\left\langle\frac{(n r)^{n+1}}{n !}\left(F^{(n)}(r n)\right)\left(i \omega-\delta^{\prime}\right) h, g\left(i \omega+\delta^{\prime}\right)\right\rangle d \omega\right| \\
& \geq \frac{m^{2}}{(1+\varepsilon)^{4}}\|h\|^{2} .
\end{aligned}
$$

This finishes the proof of Theorem 3.1. 


\section{Finite-time Admissibility}

We recall that $(A, C)$ is finite-time admissible on $(0, \tau)$ when there is a constant $M_{\tau}>0$ such that

$$
\int_{0}^{\tau}\|C T(t) x\|^{2} d t \leq M_{\tau}\|x\|^{2} \quad(x \in D(A)) .
$$

As mentioned in Section 1, the definition is independent of the choice of $\tau>0$, since if $(A, C)$ is finite-time admissible on $(0, \tau)$, then

$$
\begin{aligned}
\int_{0}^{n \tau}\|C T(t) x\|^{2} d t & =\sum_{k=0}^{n-1} \int_{0}^{\tau}\|C T(s) T(k \tau) x\|^{2} d s \\
& \leq M_{\tau} \sum_{k=0}^{n-1}\|T(k \tau)\|^{2}\|x\|^{2} \\
& \leq M_{\tau} \sum_{k=0}^{n-1}\|T(\tau)\|^{2 k}\|x\|^{2} .
\end{aligned}
$$

We conclude that, if $(A, C)$ is finite-time admissible for some $\tau>0$, then there exist constants $\alpha, \mu$ such that

$$
\int_{0}^{z}\|C T(t) x\|^{2} d t \leq \mu^{2} e^{2 \alpha z}\|x\|^{2} \quad(x \in D(A), z>0) .
$$

In this case, we shall say that $(A, C)$ is finite-time admissible with constants $\mu, \alpha$.

If $(T(t))_{t \geq 0}$ is exponentially stable, then the above argument shows that finite-time admissibility is equivalent to infinite-time admissibility. In general we may deduce that if $(A, C)$ is finite-time admissible, then there are constants $\mu, \alpha>0$ such that for every $\sigma>0$ we have

$$
\int_{0}^{\sigma}\|C T(t) x\|^{2} d t \leq \mu^{2} e^{2 \alpha \sigma}\|x\|^{2} \quad(x \in D(A)) .
$$

As it already became apparent in the proof of Theorem 2.3, one can characterize finite-time admissibility in terms of the $\gamma_{n}$.

Proposition 4.1. Let $\mathcal{H}$ be a Hilbert space and $(T(t))_{t \geq 0}$ be a strongly continuous semigroup on $\mathcal{H}$ with generator $A$ such that $\rho(A) \supseteq \mathbb{C}_{+}$. Let $C: \mathcal{D}(A) \rightarrow Y$ be an observation operator. Then $(A, C)$ is finite-time admissible if and only if there exist $r, M, N_{0}>0$ 
such that

$$
\left\|\gamma_{n}(r n)\right\|_{\mathcal{L}\left(\mathcal{H}, L^{2}((0, \infty), Y)\right)} \leq \frac{M}{(r n)^{n+1}} \quad \text { for all } n>N_{0},
$$

where $\gamma_{n}(\lambda)(t)=(-1)^{n} \sum_{k=0}^{n} \frac{t^{k} e^{-\lambda t}}{k !} C(\lambda-A)^{-(n-k+1)}$ for $t \geq 0$.

Proof. Assuming that (13) holds, we shall show finite-time admissibility on $(0,1 / r)$. Proceeding as in the proof of Theorem 2.3 , we find that

$$
\left\|C(A+i \omega)^{-1}\left(e^{A / r}-e^{-i \omega / r}\right) h\right\|_{H^{2}\left(\mathbb{C}_{+}, Y\right)} \leq M\|h\| \quad(h \in \mathcal{H}) .
$$

Since

$\mathcal{L}\left(\chi_{[0,1 / r]} C e^{A \cdot} h\right)(z)=C(A-z)^{-1}\left(e^{A / r}-e^{z / r}\right) e^{-z / r} h \quad\left(z \in \mathbb{C}_{+}\right)$, one verifies that

$$
\left\|C(A+i \omega)^{-1}\left(e^{A / r}-e^{-i \omega / r}\right) h\right\|_{H^{2}\left(\mathbb{C}_{+}, Y\right)}^{2}=\int_{0}^{1 / r}\|C T(t) h\|^{2} d t,
$$

which finishes the proof of the first implication.

The converse may be derived as follows. With $h \in \mathcal{H}$ and $g \in$ $L^{2}((0, \infty, Y))$, we use (5) and (12) to obtain the following estimate, valid for $\lambda>\alpha$ :

$\left|\left\langle\gamma_{n}(\lambda) h, g\right\rangle\right| \leq \frac{1}{n !} \int_{z=0}^{\infty} z^{n} e^{-\lambda z}\|h\| \mu e^{\alpha z}\|g\| d z=\frac{\mu}{(\lambda-\alpha)^{n+1}}\|h\|\|g\|$.

We may now choose any $r>0$. Taking $\lambda=r n$ for $n>\alpha / r$, we obtain

$\left\|\gamma_{n}(r n)\right\|_{\mathcal{L}\left(\mathcal{H}, L^{2}((0, \infty), Y)\right)} \leq \frac{\mu}{(r n-\alpha)^{n+1}}=\frac{\mu}{(r n)^{n+1}}\left(1-\frac{\alpha}{r n}\right)^{-(n+1)}$

from which (13) follows easily for any $M>\mu e^{\alpha / r}$, since the last term tends to $e^{\alpha / r}$ as $n \rightarrow \infty$.

\section{Finite-time Exact Observability}

We recall that $(A, C)$ is $\tau$-exactly observable for some $\tau>0$ if there is a constant $m_{\tau}>0$ such that

$$
\int_{0}^{\tau}\|C T(t) x\|^{2} d t \geq m_{\tau}\|x\|^{2} \quad(x \in D(A)) .
$$

This definition does depend on the choice of $\tau$ : for example it can be verified that the left-shift semigroup on $\mathcal{H}=L^{2}(0,1)$ with $C f=f(0)$ is $\tau$-exactly observable if and only if $\tau \geq 1$. 
Here is the main result of this section.

Theorem 5.1. Let $\mathcal{H}$ be a Hilbert space and $(T(t))_{t>0}$ be a strongly continuous semigroup on $\mathcal{H}$ with generator $A$ such that $\rho(A) \supseteq \mathbb{C}_{+}$. Let $C: \mathcal{D}(A) \rightarrow Y$ be an observation operator. Let $r>0$.

(1) Suppose that $(A, C)$ is finite-time admissible with constants $\mu, \alpha$, and that there exists constants $m, \varepsilon>0$ such that for each $h \in \mathcal{H}$, there exists $n \geq N=N(\varepsilon, \alpha / r, \mu, m)$ with

$$
\left\|\gamma_{n}((1+\varepsilon) r n) h\right\| \geq \frac{1}{((1+\varepsilon) r n)^{n+1}} m\|h\| .
$$

Then $(A, C)$ is $1 / r$-exactly observable.

(2) Conversely, suppose that $(A, C)$ is $1 / r$-exactly observable. Then there exists a constant $m>0$ such that for each $h \in \mathcal{H}$, there exists $n \in \mathbb{N}$ with

$$
\left\|\gamma_{n}(r n) h\right\| \geq \frac{1}{(r n)^{n+1}} m\|h\| .
$$

Proof. (1) Let $h \in \mathcal{H}$. Choosing an appropriate $g \in L^{2}((0, \infty), Y)$ with $\|g\|=1$, we write as in the proof of Lemma 2.2

$$
\begin{aligned}
& m\|h\| \\
& \leq((1+\varepsilon) r n)^{n+1}\left\|\gamma_{n}((1+\varepsilon) r n) h\right\| \\
& =((1+\varepsilon) r n)^{n+1}\left\langle\gamma_{n}((1+\varepsilon) r n) h, g\right\rangle \\
& \leq \frac{((1+\varepsilon) r n)^{n+1}}{n !}\left|\int_{0}^{\infty} z^{n} e^{-(1+\varepsilon) r n z} d z \int_{0}^{z}\langle C T(\omega) h, g(z-\omega)\rangle_{Y} d \omega\right| \\
& \leq \frac{((1+\varepsilon) r n)^{n+1}}{n !}\left|\int_{0}^{1 / r} z^{n} e^{-(1+\varepsilon) r n z} d z \int_{0}^{z}\langle C T(\omega) h, g(z-\omega)\rangle_{Y} d \omega\right| \\
& +\frac{((1+\varepsilon) r n)^{n+1}}{n !}\left|\int_{1 / r}^{\infty} z^{n} e^{-(1+\varepsilon) r n z} d z \int_{0}^{z}\langle C T(\omega) h, g(z-\omega)\rangle_{Y} d \omega\right|
\end{aligned}
$$

Using finite-time admissibility, we find that

$$
\frac{((1+\varepsilon) r n)^{n+1}}{n !}\left|\int_{1 / r}^{\infty} z^{n} e^{-(1+\varepsilon) r n z} d z \int_{0}^{z}\langle C T(\omega) h, g(z-\omega)\rangle_{Y} d \omega\right|
$$




$$
\begin{aligned}
& \leq \frac{((1+\varepsilon) r n)^{n+1}}{n !} \int_{1 / r}^{\infty} \mu z^{n} e^{-((1+\varepsilon) r n-\alpha) z} d z \\
& \leq \mu\left(\frac{(1+\varepsilon) r n}{(1+\varepsilon) r n-\alpha}\right)^{n+1} \frac{1}{n !} \int_{(1+\varepsilon) n-\alpha / r}^{\infty} z^{n} e^{-z} d z \\
& \lesssim \mu e^{\alpha / r} \frac{1}{n !} \int_{(1+\varepsilon) n-\alpha / r}^{\infty} z^{n} e^{-z} d z
\end{aligned}
$$

for $n r>\alpha$. Notice that $\chi_{[0, \infty)} \frac{1}{n !} z^{n} e^{-z}$ is the $(n+1)$ st convolution power of the density function of the exponential distribution $\chi_{[0, \infty)} e^{-z}$. It follows from the Central Limit Theorem that $\frac{1}{n !} \int_{(1+\varepsilon) n-\alpha / r}^{\infty} z^{n} e^{-z} d z \rightarrow 0$ as $n \rightarrow \infty$. Therefore, there exists $N \in \mathbb{N}$, only depending on $\epsilon, m, \alpha / r$ and $\mu$, such that

$$
\left\|\gamma_{n}((1+\varepsilon) r n) h\right\| \geq \frac{1}{((1+\varepsilon) r n)^{n+1}} m\|h\| \text { for some } n \geq N
$$

implies

$$
\begin{aligned}
\mid \int_{0}^{1 / r} z^{n} e^{-(1+\varepsilon) r n z} d z \int_{0}^{z}\langle C T(\omega) h, & g(z-\omega)\rangle_{Y} d \omega \mid \\
& \geq \frac{n !}{((1+\varepsilon) r n)^{n+1}} \frac{m}{2}\|h\|
\end{aligned}
$$

and therefore

$$
\begin{aligned}
& \frac{m}{2}\|h\| \\
& \leq \frac{((1+\varepsilon) r n)^{n+1}}{n !} \int_{0}^{1 / r} z^{n} e^{-(1+\varepsilon) r n z} d z \int_{0}^{1 / r}\|C T(\omega) h\|\|g(z-\omega)\| d \omega \\
& \leq \frac{((1+\varepsilon) r n)^{n+1}}{n !} \int_{0}^{\infty} z^{n} e^{-(1+\varepsilon) r n z} d z\left(\int_{0}^{1 / r}\|C T(\omega) h\|^{2} d \omega\right)^{1 / 2} \\
& =\left(\int_{0}^{1 / r}\|C T(\omega) h\|^{2} d \omega\right)^{1 / 2} .
\end{aligned}
$$

Part (2) of the theorem is proved exactly as in the forward direction of Theorem 3.1. 


\section{Boundedness of Vector Hankel Operators}

It is possible to use Theorem 2.3 to give a characterization of the boundedness of vector Hankel operators, using the right-shift semigroup on $L^{2}(0, \infty)$ as in $[6,7,8]$. However, one can derive the analogous results by a more direct argument, and this is what we do now.

Define $k_{z}(s)=\frac{1}{(s+\bar{z})}$, the reproducing kernel for $H^{2}\left(\mathbb{C}_{+}\right)$, which satisfies

$$
f(z)=\frac{1}{2 \pi} \int_{-\infty}^{\infty} f(i w) \overline{k_{z}(i w)} d w, \quad \text { for } f \in H^{2}\left(\mathbb{C}_{+}\right) \text {and } z \in \mathbb{C}_{+} .
$$

For $n \in \mathbb{N}$, let $k_{z}^{(n)}$ denote the $n$-fold derivative of $k_{z}$,

$$
k_{z}^{(n)}(s)=(-1)^{n} n !\left(\frac{1}{s+\bar{z}}\right)^{n+1}=(-1)^{n} n ! k_{z}^{n+1} .
$$

This is the Laplace transform of the function

$$
(-1)^{n} \mathfrak{k}_{z}^{(n)}: t \mapsto(-1)^{n} t^{n} e^{-\bar{z} t} .
$$

A densely-defined Hankel integral operator $\Gamma_{h}: L^{2}((0, \infty), \mathcal{K}) \rightarrow$ $L^{2}((0, \infty), Y)$ is given by

$$
u \mapsto \Gamma_{h} u=\int_{0}^{\infty} h(\tau+w) u(\tau) d w .
$$

Here, $h:(0, \infty) \rightarrow \mathcal{L}(\mathcal{K}, Y)$ is assumed to be measurable. If $\mathcal{K}=Y$ then $\Gamma_{h}$ has a natural interpretation whenever $h$ is scalar-valued. Moreover, by the Paley-Wiener theorem there is a unitarily equivalent (Hankel) operator defined between $H^{2}\left(\mathbb{C}_{+}, \mathcal{K}\right)$ and $H^{2}\left(\mathbb{C}_{-}, Y\right)$, given by a multiplication followed by an orthogonal projection.

Now let $\Gamma_{\mathfrak{k}_{\lambda}^{(n)}}$ be defined using the kernels above, and let $\tilde{\Gamma}_{\mathfrak{k}_{\lambda}^{(n)}}=$ $\frac{(\operatorname{Re} \lambda)^{n+1}}{n !} \Gamma_{\mathfrak{k}_{\lambda}^{(n)}}$ for $n \in \mathbb{N}, \lambda \in \mathbb{C}_{+}$. The set

$$
\mathcal{M}=\left\{\tilde{\Gamma}_{\mathfrak{k}_{\lambda}^{(n)}}: n \in \mathbb{N}, \lambda \in \mathbb{C}_{+}\right\}
$$

has SOT cluster points, some of which we investigate below.

Lemma 6.1. For $n \in \mathbb{N}$ and $r>0$, let $P_{r, n}=(-1)^{n} \tilde{\Gamma}_{\mathfrak{k}_{r n}^{(n)}}$. Let $e_{r}: \mathbb{C}_{+} \rightarrow \mathbb{D}$ be defined by $w \mapsto e^{-r w}$. Then for each $r>0$, the sequence $\left(P_{r, n}\right)_{n \in \mathbb{N}}$ converges strongly to the Hankel operator $\Gamma_{e_{r}}$. 
Proof. Notice that for each $r>0$,

$$
(-1)^{n} \frac{(r n)^{n+1}}{n !} k_{r n}^{(n)}(w)=\left(\frac{r n}{(r n+w)}\right)^{n+1} \quad\left(w \in \mathbb{C}_{+}\right),
$$

which converges for $n \rightarrow \infty$ uniformly on bounded sets in $\mathbb{C}_{+}$to $e^{-w / r}$. It follows that for any $f \in H^{2}\left(\mathbb{C}_{+}, \mathcal{K}\right),(-1)^{n} \frac{(r n)^{n+1}}{n !} k_{r n}^{(n)} f \rightarrow$ $e^{-\cdot / r} f$ in norm, and we have strong convergence of the corresponding multiplication operators. Thus, $P_{r, n} \rightarrow \Gamma_{e_{1 / r}}$ in SOT as $n \rightarrow \infty$.

We can use this to obtain an elementary proof for a boundedness theorem for vector Hankel operators, which is also a consequence of Theorem 2.3.

Theorem 6.2. Let $\Gamma_{h}: L^{2}((0, \infty), \mathcal{K}) \rightarrow L^{2}((0, \infty), Y)$ be a densely defined Hankel operator given by

$$
u \mapsto \Gamma_{h} u=\int_{0}^{\infty} h(\tau+w) u(\tau) d w,
$$

where $h:(0, \infty) \rightarrow \mathcal{L}(\mathcal{K}, Y)$ is measurable. Then $\Gamma_{h}$ defines a bounded linear operator on $L^{2}((0, \infty), \mathcal{K})$ if and only if there exists a constant $M>0$ such that for each $\lambda>0$, each $n \in \mathbb{N}$ and each $\mathrm{y} \in L^{2}((0, \infty), Y)$,

$$
\left\|\Gamma_{h}^{*} \Gamma_{\mathfrak{k}_{\lambda}^{(n)}} \mathrm{y}\right\| \leq M\|\mathrm{y}\| \frac{n !}{\lambda^{n+1}},
$$

that is, $\Gamma_{h}^{*}$ acts boundedly on the images of the scalar Hankel operators $\Gamma_{\mathfrak{k}_{\lambda}^{(n)}}$ with uniform bound.

Proof. Notice that the Hankel operator $\Gamma_{e_{r}}$ on $H^{2}\left(\mathbb{C}_{+}\right)$is unitarily equivalent via the Laplace transform to the translation-reflection operator

$$
T_{r}: L^{2}(0, \infty) \rightarrow L^{2}(0, \infty), \quad T_{r} f(t)=\left\{\begin{array}{cc}
f(r-t) & \text { if } r>t \\
0 & \text { otherwise }
\end{array}\right.
$$

By the preceding lemma, the conditions of the theorem imply that $\left\|T_{r} \Gamma_{h}\right\|=\left\|\Gamma_{h}^{*} T_{r}\right\| \leq M$ for all $r>0$. Since $\bigcup_{r>0}$ Range $T_{r}$ contains the compactly supported functions in $L^{2}(0, \infty)$ and $\lim _{r \rightarrow \infty}\left\|T_{r} f\right\|=$ $\|f\|$ for all $f \in L^{2}(0, \infty)$, it follows that $\Gamma_{h}$ is bounded, and $\left\|\Gamma_{h}\right\| \leq M$. 


\section{Operator BMO and the Dual of $H^{1}\left(\mathbb{C}_{+}, S_{1}\right)$}

Let $\mathcal{K}$ be a separable infinite-dimensional Hilbert space, and let $S_{1}$ denote the ideal of trace-class operators in $\mathcal{L}(\mathcal{K})$. The following fact is a well-known consequence of Sarason's factorization theorem $H^{1}\left(\mathbb{C}_{+}, S_{1}\right)=H^{2}(\mathcal{K}) \hat{\otimes} H^{2}(\mathcal{K})[9]$.

Proposition 7.1. Let $\mathcal{B}$ be an operator-valued measure on $i \mathbb{R}$ such that $\int_{i \mathbb{R}} d \mathcal{B}^{*} \mathcal{B}$ converges in the WOT-topology and such that

$$
\int_{-\infty}^{\infty} e^{i \omega t} d \mathcal{B}(i \omega)=0 \quad \text { for a.e. } t<0 .
$$

Then $\mathcal{B}$ gives rise to a bounded vector Hankel operator $\Gamma_{\mathcal{B}}$ on $H^{2}\left(\mathbb{C}_{+}, \mathcal{K}\right)$; that is, the densely defined sesquilinear map

$$
\begin{gathered}
H^{2}\left(\mathbb{C}_{+}, \mathcal{K}\right) \times H^{2}\left(\mathbb{C}_{-}, \mathcal{K}\right) \rightarrow \mathbb{C}, \\
(f, g) \mapsto\left\langle\Gamma_{\mathcal{B}} f, g\right\rangle=\int_{-\infty}^{\infty}\langle d \mathcal{B}(i \omega) f(i \omega), g(i \omega)\rangle
\end{gathered}
$$

extends to a bounded sesquilinear form on $H^{2}\left(\mathbb{C}_{+}, \mathcal{K}\right) \times H^{2}\left(\mathbb{C}_{-}, \mathcal{K}\right)$ if and only if $\mathcal{B}$ defines a bounded linear functional on $H^{1}\left(\mathbb{C}_{+}, S_{1}\right)$ via

$$
F \mapsto \int_{-\infty}^{\infty} \operatorname{trace}(d \mathcal{B}(i \omega) F(i \omega)) .
$$

In this case, $\left\|\Gamma_{\mathcal{B}}\right\|=\|\mathcal{B}\|_{H^{1}\left(\mathbb{C}_{+}, S_{1}\right)^{*}}$.

We write $H^{1}\left(\mathbb{C}_{-}, S_{1}\right)$ for the space $\left\{F: \mathbb{C}_{+} \rightarrow S_{1} \mid F^{*} \in H^{1}\left(\mathbb{C}_{+}, S_{1}\right)\right\}$ and $H_{r e}^{1}\left(i \mathbb{R}, S_{1}\right)$ for the space $H^{1}\left(\mathbb{C}_{+}, S_{1}\right)+H^{1}\left(\mathbb{C}_{-}, S_{1}\right)$ with the norm $\|H\|_{H^{1}}=\|F+G\|_{H^{1}}=\|F\|_{H^{1}\left(\mathbb{C}_{+}, S_{1}\right)}+\left\|G^{*}\right\|_{H^{1}\left(\mathbb{C}_{+}, S_{1}\right)}$ for $H=F+G$ with $F \in H^{1}\left(\mathbb{C}_{+}, S_{1}\right)$ and $G \in H^{1}\left(\mathbb{C}_{-}, S_{1}\right)$.

For an operator-valued function $B: i \mathbb{R} \rightarrow \mathcal{L}(\mathcal{K})$ such that $B^{*} B$ is WOT integrable on $i \mathbb{R}$, we can define its analytic and anti-analytic parts $B^{+}$and $B^{-}$, given by $B^{+} e=P^{+} B e, B^{-} e=P^{-} B e$ for $e \in \mathcal{K}$, where $P^{+}: L^{2}(i \mathbb{R}, \mathcal{K}) \rightarrow H^{2}\left(\mathbb{C}_{+}, \mathcal{K}\right)$ denotes the Riesz projection and $P^{-}$the corresponding orthogonal projection.

One sees easily that such an operator function $B$ defines a bounded linear functional on $H_{r e}^{1}(i \mathbb{R}, \mathcal{K})$ if and only if both $B^{-}$and $\left(B^{+}\right)^{*}$ are in the dual of $H^{1}\left(\mathbb{C}_{+}, S_{1}\right)$.

We obtain the following consequence of Theorem 6.2. 
Theorem 7.2. Let $B: i \mathbb{R} \rightarrow \mathcal{L}(\mathcal{K})$ be an operator-valued function such that $B^{*} B$ is WOT integrable on $i \mathbb{R}$. Then $B$ defines a bounded linear functional on $H_{r e}^{1}\left(i \mathbb{R}, S_{1}\right)$ if and only if there exists a constant $C>0$ such that

$$
\left|\int_{-\infty}^{\infty} \int_{-\infty}^{\infty} \operatorname{trace}\left((B(i \omega)-B(i t)) \phi_{s}^{(n)}(i \omega, i t) f(i \omega) \otimes g(i t)\right) d \omega d t\right|
$$

is at most $C / s^{n+1}$ for all $s \in \mathbb{R}_{+}, f \in L^{2}(i \mathbb{R}, \mathcal{K}), g \in L^{2}(i \mathbb{R}, \mathcal{K})$ and $n \in \mathbb{N}_{0}$, where

$$
\phi_{s}^{(n)}(i w, i t)=\frac{1}{(i \omega+s)(i t+s)} \sum_{k=0}^{n} \frac{1}{(i \omega+s)^{k}(i t+s)^{n-k}}
$$

for $s \in \mathbb{C}_{+}, t, w \in \mathbb{R}$, and $n \in \mathbb{N}_{0}$.

Remark. Note that (14) is a bounded mean oscillation-type condition. Recall that one of the characterizations of the scalar BMO space $\mathrm{BMO}(i \mathbb{R})$, the dual of the scalar space $H_{r e}^{1}(i \mathbb{R})$, is that $b$ belongs to $B M O(i \mathbb{R})$ if and only if there exists a constant $C>0$ such that

$$
\begin{aligned}
& \int_{-\infty}^{\infty} \int_{-\infty}^{\infty}|b(i \omega)-b(i t)|^{2}\left|\phi_{s}^{(0)}(i w, i t)\right|^{2} d t d \omega \\
= & \frac{1}{\pi^{2}(\operatorname{Re} s)^{2}} \int_{-\infty}^{\infty} \int_{-\infty}^{\infty}|b(i \omega)-b(i t)|^{2} p_{s}(t) p_{s}(\omega) d t d \omega \leq \frac{C}{(\operatorname{Re} s)^{2}}
\end{aligned}
$$

for all $s \in \mathbb{C}_{+}$and $b(i t)$ satisfies a certain decay condition as $|t| \rightarrow \infty$. (For further information on scalar BMO, see e.g. [2].) For $n=0$, we know that the condition that

$$
\left|\int_{-\infty}^{\infty} \int_{-\infty}^{\infty} \operatorname{trace}\left((B(i \omega)-B(i t)) f(i \omega) \otimes g(i t) \frac{1}{(i \omega+s)(i t+s)}\right) d \omega d t\right|
$$

is at most $C / s$ for all $s \in \mathbb{C}_{+}, f \in H^{2}\left(\mathbb{C}_{+}, \mathcal{K}\right)$ and $g \in H^{2}\left(\mathbb{C}_{+}, \mathcal{K}\right)$ is one characterization of the so-called adjoint strong operator $B M O$ condition: $B^{*} e \in B M O(i \mathbb{R}, \mathcal{K})$ with uniformly bounded norm for $e \in \mathcal{K},\|e\| \leq 1$. This condition is general not sufficient for $\mathcal{B}$ to be in $H^{1}\left(\mathbb{C}_{+}, S_{1}\right)$, but corresponds to the resolvent condition (2). 
Admissibility and Exact Observability of Observation Operators 37

Alternatively, for $B$ anti-analytic, the condition in (14) simplifies to

$$
\begin{aligned}
& \left|\int_{-\infty}^{\infty} \int_{-\infty}^{\infty} \operatorname{trace}\left(B(i \omega) \phi_{s}^{(n)}(i \omega, i t) f(i \omega) \otimes g(i t)\right) d \omega d t\right| \\
& \quad \leq C \frac{1}{s^{n+1}} \quad \text { for all } s \in \mathbb{R}_{+}, f, g \in H^{2}\left(\mathbb{C}_{+}, \mathcal{K}\right), n \in \mathbb{N}_{0}
\end{aligned}
$$

and can be understood as a test function condition on certain elements of $H^{1}\left(\mathbb{C}_{+}, S_{1}\right)$, namely those of the form

$$
z \mapsto \int_{-\infty}^{\infty} \phi_{s}^{(n)}(z, i t) f(z) \otimes g(i t) d t=\left(f \otimes \Gamma_{\mathfrak{k}_{s}^{(n)}}^{*} g\right)(z)
$$

with the notation of the previous section.

Proof of 7.2. To prove that (14) is necessary, note first that the function $\bar{\phi}_{s}^{(n)}(i \omega, i t)=\left(\frac{d}{d s}\right)^{n} k_{s}(i t) k(i \omega)$ is up to a factor $(-1)^{n}$ the integral kernel for the Hankel operator $\Gamma_{\mathfrak{k}_{s}^{(n)}}$, taken as an operator $L^{2}(i \mathbb{R}, \mathcal{K}) \rightarrow L^{2}(i \mathbb{R}, \mathcal{K})$ from the previous section. This is easily seen with $(-1)^{n} \Gamma_{\mathfrak{k}_{s}^{(n)}}=\left(\frac{d}{d s}\right)^{n} \Gamma_{k_{s}}=\left(\frac{d}{d s}\right)^{n} k_{s} \otimes k_{s}$. The operator $\Gamma_{\mathfrak{k}_{s}^{(n)}}$ is bounded on $L^{2}(i \mathbb{R}, \mathcal{K})$ with norm less or equal to $\left\|k_{s}^{(n)}\right\|_{\infty}=n !\left(\frac{1}{s}\right)^{n+1}$.

Suppose first that $B$ is anti-analytic and split $f, g$ into analytic and anti-analytic parts, $f=f^{+}+f^{-}, g=g^{+}+g^{-}$. Note that

$$
\begin{aligned}
& \int_{-\infty}^{\infty} \int_{-\infty}^{\infty} \operatorname{trace}\left((B(i \omega)-B(i t)) \phi_{s}^{(n)}(i \omega, i t) f^{+}(i \omega) \otimes g^{-}(i t)\right) d t d \omega \\
& =\int_{-\infty}^{\infty} \int_{-\infty}^{\infty} \operatorname{trace}\left(B(i \omega) \phi_{s}^{(n)}(i \omega, i t) f^{+}(i \omega) \otimes g^{-}(i t)\right) d t d \omega=0
\end{aligned}
$$

and

$$
\begin{gathered}
\int_{-\infty}^{\infty} \int_{-\infty}^{\infty} \operatorname{trace}\left((B(i \omega)-B(i t)) \phi_{s}^{(n)}(i \omega, i t) f^{-}(i \omega) \otimes g^{+}(i t)\right) d t d \omega \\
=\left(\frac{d}{d s}\right)^{n} \int_{-\infty}^{\infty} \int_{-\infty}^{\infty} \operatorname{trace}\left((B(i \omega)-B(i t)) k_{s}(i t) k_{s}(i \omega) \times\right. \\
\left.\times f^{-}(i \omega) \otimes g^{+}(i t)\right) d t d \omega \\
=\left(\frac{d}{d s}\right)^{n} \operatorname{trace}((B(\bar{s})-B(\bar{s})) f(\bar{s}) \otimes g(\bar{s}))=0
\end{gathered}
$$


Furthermore,

$$
\begin{array}{r}
\int_{-\infty}^{\infty} \int_{-\infty}^{\infty} \operatorname{trace}\left((B(i \omega)-B(i t)) \phi_{s}^{(n)}(i \omega, i t) f^{+}(i \omega) \otimes g^{+}(i t)\right) d t d \omega \\
=\int_{-\infty}^{\infty} \int_{-\infty}^{\infty} \operatorname{trace}\left(B(i \omega) \phi_{s}^{(n)} f^{+}(i \omega) \otimes g^{+}(i t)\right) d t d \omega \\
\quad=\left\langle B f, \int_{-\infty}^{\infty} \bar{\phi}_{s}^{(n)}(i \omega, i t) g^{+}(i t) d t\right\rangle=\left\langle\Gamma_{B} f^{+}, \Gamma_{\mathfrak{k}_{s}^{(n)}} g^{+}\right\rangle
\end{array}
$$

and

$$
\begin{gathered}
\int_{-\infty}^{\infty} \int_{-\infty}^{\infty} \operatorname{trace}\left((B(i \omega)-B(i t)) \phi_{s}^{(n)}(i \omega, i t) f^{-}(i \omega) \otimes g^{-}(i t)\right) d t d \omega \\
=-\int_{-\infty}^{\infty} \int_{-\infty}^{\infty} \operatorname{trace}\left(B(i t) \phi_{s}^{(n)} f^{-}(i \omega) \otimes g^{-}(i t)\right) d t d \omega \\
=-\left\langle\Gamma_{\mathfrak{k}_{s}^{(n)}}^{*} f^{-}, \Gamma_{B}^{*} g^{-}\right\rangle .
\end{gathered}
$$

This yields the required estimates. A similar but simpler argument covers the case that $B$ is analytic.

To prove the sufficiency of (14), suppose first that $B$ is antianalytic and take $f, g \in H^{2}\left(\mathbb{C}_{+}, \mathcal{K}\right)$. Let $\mathcal{H}=H^{2}\left(\mathbb{C}_{+}, \mathcal{K}\right)$, let $(T(t))_{t \geq 0}$ be the semigroup on $\mathcal{H}$ induced by the right shift semigroup on $L^{2}((0, \infty) ; \mathcal{K})$ via Laplace transform, and let $C: D(A) \rightarrow \mathcal{K}$ be given by $f \mapsto \int_{-\infty}^{\infty} B(i \omega) f(i \omega) d \omega$. Then, with the notation of (3), we have that

$$
F(\lambda) f(z)=\int_{-\infty}^{\infty} B(i \omega) f(i \omega) \frac{1}{(i \omega+\lambda)(z+\lambda)} d \omega
$$

and

$$
\begin{aligned}
& \left(F^{(n)}(\lambda) f\right)(z) \\
& \quad=(-1)^{n} n ! \int_{-\infty}^{\infty} B(i \omega) f(i \omega) \sum_{k=0}^{n} \frac{1}{(i \omega+\lambda)^{k+1}(z+\lambda)^{n+1-k}} d \omega .
\end{aligned}
$$

The result follows now from Theorem 2.3. The case of general $B$ follows easily.

\section{REFERENCES}

[1] M.-C. Gao and J.-C. Hou, The infinite-time admissibility of observation operators and operator Lyapunov equations, Int. Eq. Oper. Th. 35 (1999), 53-64. 
[2] J.B. Garnett, Bounded Analytic Functions, Academic Press, New York, 1981.

[3] P. Grabowski and F.M. Callier, Admissible observation operators. Semigroup criteria of admissibility, Int. Eq. Oper. Th. 25 (1996), 182-198.

[4] B. Jacob and J.R. Partington, The Weiss conjecture on admissibility of observation operators for contraction semigroups, Int. Eq. Oper. Th. 40 (2001), 231-243.

[5] B. Jacob and J.R. Partington, Admissibility of control and observation operators for semigroups: a survey, In: Current Trends in Operator Theory and its Applications, Proceedings of IWOTA 2002, J.A. Ball, J.W. Helton, M. Klaus and L. Rodman (eds.), Operator Theory: Advances and Applications 149, Birkhäuser (2004), 199-221.

[6] B. Jacob, J.R. Partington and S. Pott, Admissible and weakly admissible observation operators for the right shift semigroup, Proc. Edinburgh Math. Soc. 45 (2002), 353-362.

[7] B. Jacob, J.R. Partington and S. Pott, Conditions for admissibility of observation operators and boundedness of Hankel operators, Int. Eq. Oper. Th. 47 (2003), 315-338.

[8] J.R. Partington and G. Weiss, Admissible observation operators for the right shift semigroup, Mathematics of Control, Signals and Systems 13 (2000), 179-192.

[9] D. Sarason, Generalized interpolation in $H^{\infty}$, Trans. Amer. Math. Soc. 127 (1967), 179-203.

[10] O. Staffans, Well-Posed Linear Systems, Cambridge University Press, 2005.

[11] G. Weiss, Two conjectures on the admissibility of control operators, In: Estimation and Control of Distributed Parameter Systems, W. Desch, F. Kappel (eds.), Birkhäuser (1991), 367-378.

[12] G. Weiss, A powerful generalization of the Carleson measure theorem?, In: Open problems in Mathematical Systems Theory and Control, V. Blondel, E. Sontag, M. Vidyasagar and J. Willems (eds.), Springer-Verlag, 1998, $267-272$.

[13] H. Zwart, B. Jacob and O. Staffans, Weak admissibility does not imply admissibility for analytic semigroups, Systems Control Lett. 48 (2003), 341350 .

Jonathan R. Partington,

School of Mathematics,

University of Leeds,

Leeds LS2 9JT, U.K.

J.R.Partington@leeds.ac.uk

\author{
Sandra Pott, \\ Department of Mathematics, \\ University of Glasgow, \\ University Gardens, \\ Glasgow G12 8QW, U.K. \\ sp@maths.gla.ac.uk
}

